\title{
THE THEORY OF CURVED, MULTI-CELL BOX GIRDER BRIDGES UNDER CONSIDERATION OF CROSS-SECTIONAL DISTORTION
}

\author{
By Tsuneo USUKI*
}

\begin{abstract}
The theory of curved, thin-walled, multi-cellular beams and a method of determining the stresses and deformation, including warping, are presented. The equations governing small displacements in the linear elastic range are derived using the principle of virtual work. The solution includes the distortion of the cross-section, the influence of shear deformation, and the variation of longitudinal fibre curvature.

Keywords : Curved girder, multi-cell box girder, distortion
\end{abstract}

\section{INTRODUCTION}

The theory of torsion bending is generally used for the analysis of curved box girder bridges on the assumption that the shape of the cross-section does not change. Based on this assumption, research has been performed on the effects of variation of longitudinal fibre curvature, i. e., Refs. 1) 5). On the other hand, the methods for analyzing curved bridges with considertion of cross-sectional distortion can, when the subject is limited to box girder, be classified into beam theory, the generalized coordinate method, folded plate theory, the finite element method, the finite strip method, combined use of the finite element method and the finite strip method, combined use of the finite strip method and the finite difference method, combination of the finite element method and the generalized coordinate method, etc.

In this research, the beam thory is used with the prime object of smoothly combining the theory of torsion bending and the cross-sectional distortion of curved beams. For summaries of analysis methods other than the beam theory, refer to Refs.6) and 7).

Research dealing with the cross-sectional distortion of curved box girders using the beam theory is rare compared with that for straight box girders, and to the best of the author's knowledge, it is limited to the research report by Dabrowski ${ }^{8)}$, the theory evolved therefrom based on the equations obtained from the research $^{9)}$, and the theory induced from a similar position to Dabrowski ${ }^{10)}$. In beams with a square cross-section, the warping of torsion and that of distortion are coupled. However, in Ref. 8) an analogous equation to the beam on elastic foundation is derived by disregarding this coupling. Although this equation is frequntly used in the designing of intermediate diaphragms of steel box girder bridges because of its simplicity, it is quetionable whether it can be applied to single- and multi-cell box girder bridges with a

* Member of JSCE, Dr.-Ing., Dr. Eng., Associate Professor, Department of Civil Engineering, Science University of Tokyo (2641, Yamazaki, Noda-shi, Chiba-ken) 
large curvature.

The object of this report is to derive governing equations of the curved multi-cell box girder bridges with deformable cross-section in parallel with the theory of torsion bending in order to equally evaluate the coupling of torsion and distortion of the cross-section. Although this research is an extension of the author's report on straight multi-cell box girder bridges ${ }^{11)}$ into curved multi-cell box girder bridges, as in that report, an equation is derived using the principle of virtual work, and the cross-sectional distortion pattern is extracted by orthogonalization of the warping resistance marix. The deformation caused by a secondary shearing stress that equilibrates with the warping stress of thin-walled elements is called the secondary shear deformation. The effects of this deformation are particularly remarkable in the torsion bending phenomenon of a closed cross-sectional member. Therefore, this report also takes into account the effects of this deformation in order to preserve the strictness and conformability of the theory.

Research has been performed on the distortion of curved, thin-walled, open cross-sectional members using the beam theory by making kinematic consideration of each plate, i. e., Refs. 12) and 13). In these open cross-sectional members, there are no problems of coupling of warpings of torsion and distortion, and the secondary shear deformation is not taken into consideration because of its small effects.

\section{COORDINATE SYSTEMS AND ASSUMPTIONS}

A cylindrical coordinate system $(\delta, \rho, \theta)$ is used as shown in Fig. 1 and axis of the cylinder $\delta$ coincides with the center of curvature of the beam. For any cross section of the beam, a rectangular coordinate system $(x, y, z)$ is used. The neutral axis formed by connecting neutral point $\mathrm{N}$ of the cross-section is called the $x$ axis, and the principal axes $y$ and $z$ are determined at right angles therefrom. The angle between the $\rho$ axis and the $y$ axis is assumed to be $\beta$. In addition to this coordinate system, a coordinate $s$ that passes the centerline of wall thickness and coordinate $n$ at right angle to the coordinate $s$ are also used. The origin of the coordinate $s$ coincides with the neutral point $\mathrm{N}$ of the cross-section, and this point is connected to an optional position on the plate constituting a cross-section with an imaginary plate of zero thickness ${ }^{4}$.

Basic assumptions used in this report are as follows :

a) The cross-section consists of plates, the centerline of the wall thicness of which forms a straight line.

b) The plate thickness $t$ is small in comparison to the plate width $b$.

c) The structural material satisfies Hooke's Law.

d) The extensional strain in the direction of the coordinate $s$ is zero.

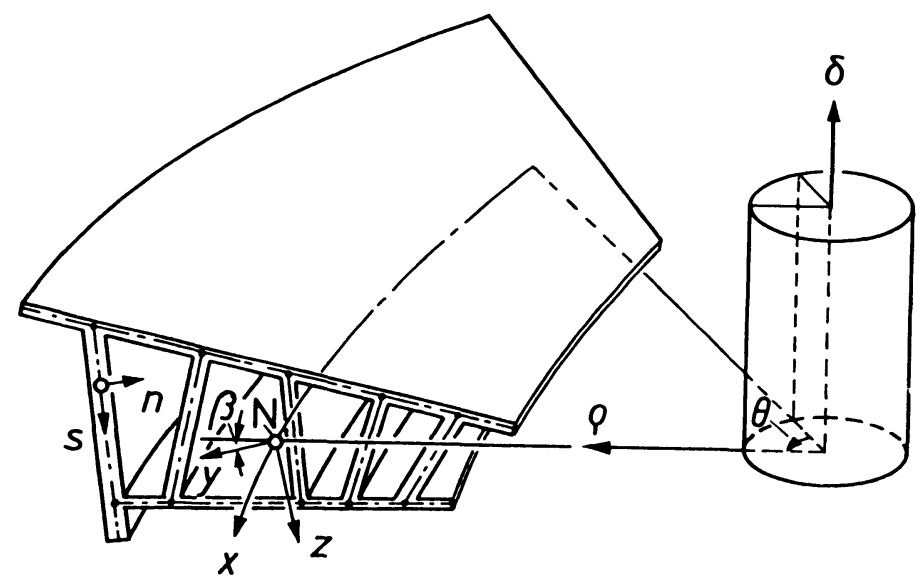

Fig. 1 Coordinate System. 
e) Displacements of the positions of the neutral point $\mathrm{N}$ and the shear centre $\mathrm{M}$ when distortion takes place are disregarded as they are extremely small.

f) In the calculation of displacement due to distortion the system is assumed to be a folded plate structure with simply connected edges.

g) In the calculation of lateral bending moment the system is assumed to be a folded plate structure with rigidly connected edges.

h) In the estimation of normal stress the displacement in the direction of the $\rho$ axis due to distortion is disregarded as it is extremly small.

\section{BASIC EQUATIONS}

(1) Strain and displacement Assuming that the displacement components in the direction of the $\rho$, $\theta, y, z$ axis at an arbitrary point $\mathrm{A}$ on the centerline of the wall thickness are $r, u, v, w$, respectively, the following relationship exists between displacements and strains at that point :

$$
\left.\begin{array}{l}
\varepsilon_{\theta}=\frac{1}{\rho} \frac{\partial u}{\partial \theta}+\frac{r}{\rho}, \quad \varepsilon_{y}=\frac{\partial v}{\partial y}, \quad \varepsilon_{z}=\frac{\partial w}{\partial z} \\
\gamma_{\theta y}=\frac{1}{\rho} \frac{\partial v}{\partial \theta}+\rho \frac{\partial}{\partial y}\left(\frac{u}{\rho}\right), \quad \gamma_{y z}=\frac{\partial w}{\partial y}+\frac{\partial v}{\partial z}, \quad \gamma_{z \theta}=\frac{1}{\rho} \frac{\partial w}{\partial \theta}+\rho \frac{\partial}{\partial z}\left(\frac{u}{\rho}\right)
\end{array}\right\}
$$

Also, if the displacement in the direction of coordinate $s$ at the point A can be expressed as $f$, the shearing strain on $\theta-s$ plane would become as follows :

$$
\gamma_{\theta s}=\frac{1}{\rho} \frac{\partial f}{\partial \theta}+\rho \frac{\partial}{\partial s}\left(\frac{u}{\rho}\right)
$$

The infinitesimal length $d x$ can be obtained by the following equation using the distance $R_{N}$ between the centre of curvature and the neutral point $\mathrm{N}$ of the cross-section :

$d x=R_{N} d \theta$

Using Eq. (3), Eqs. (1) and (2) can be rewritten, and by substituting $\theta$ for $x$, the following is obtained :

$$
\left.\begin{array}{l}
\varepsilon_{x}=\frac{\partial}{\partial x}\left(\frac{R_{N}}{\rho} u\right)+\frac{r}{\rho}, \quad \varepsilon_{y}=\frac{\partial v}{\partial y}, \quad \varepsilon_{z}=\frac{\partial w}{\partial z} \\
\frac{R_{N}}{\rho} \gamma_{x y}=\frac{\partial}{\partial x}\left[\left(\frac{R_{N}}{\rho}\right)^{2} v\right]+\frac{\partial}{\partial y}\left(\frac{R_{N}}{\rho} u\right), \quad \gamma_{y z}=\frac{\partial w}{\partial y}+\frac{\partial v}{\partial z}, \quad \frac{R_{N}}{\rho} \gamma_{z x}=\frac{\partial}{\partial x}\left[\left(\frac{R_{N}}{\rho}\right)^{2} w\right]+\frac{\partial}{\partial z}\left(\frac{R_{N}}{\rho} u\right)
\end{array}\right\}
$$

$$
\frac{R_{N}}{\rho} \gamma_{x s}=\frac{\partial}{\partial x}\left[\left(\frac{R_{N}}{\rho}\right)^{2} f\right]+\frac{\partial}{\partial s}\left(\frac{R_{N}}{\rho} u\right)
$$

Although the shearing strain due to simple torsion, that is the primary shearing strain, is considered in these equations, the strain due to secondary shearing stress is not considered. Following the conventional beam theory, to take this secondary shearing strain into account, in this report, the in-plane displacement component is fixed, and only the out-of-plane displacement component $u$, is reduced to $u_{1}$. In other words, if the reduction in warping due to secondary shearing strain is expressed as $u_{2}$, it could be derived from Fig. 2 as follows :

$u_{1}=u-u_{2}$

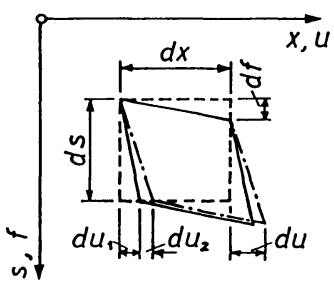

Fig. 2 Shearing strain.

If the primary shearing strain and the secondary shearing strain can be expressed as $\gamma_{x s, p r}$ and $\gamma_{x s, s e}$, respectively, the total shearing strain $\gamma_{x s}$ would be expressed as follows :

$\gamma_{x s}=\gamma_{x s, p r}+\gamma_{x s, s e}$

Using Eqs. (6) and (7), Eqs. (4) and (5) can be rewritten as follows : 


$$
\begin{aligned}
& \varepsilon_{x}=\frac{\partial}{\partial x}\left(\frac{R_{N}}{\rho} u_{1}\right)+\frac{r}{\rho}, \quad \varepsilon_{y}=\frac{\partial v}{\partial y}, \quad \varepsilon_{z}=\frac{\partial w}{\partial z} \\
& \frac{R_{N}}{\rho} \gamma_{x y}=\frac{\partial}{\partial x}\left[\left(\frac{R_{N}}{\rho}\right)^{2} v\right]+\frac{\partial}{\partial y}\left(\frac{R_{N}}{\rho} u_{1}\right), \quad \gamma_{y z}=\frac{\partial w}{\partial y}+\frac{\partial v}{\partial z}, \quad \frac{R_{N}}{\rho} \gamma_{z x}=\frac{\partial}{\partial x}\left[\left(\frac{R_{N}}{\rho}\right)^{2} w\right]+\frac{\partial}{\partial z}\left(\frac{R_{N}}{\rho} u_{1}\right)
\end{aligned}
$$

\section{(2) Basic conditions}

a) Condition of strain

If the elastic beam deforms, strains occurring in the beam can be expressed as a sum of the rigid components and distortional componets of the cross-section. By distinguishing them with suffixes $R$ and $D$, respectively, the assumption for a rigid cross-section would be :

$\left(\varepsilon_{y}\right)_{R}=0, \quad\left(\varepsilon_{z}\right)_{R}=0, \quad\left(\gamma_{y z}\right)_{R}=0$.

On the other hand, the strain $\left(\varepsilon_{y}\right)_{D}, \quad\left(\varepsilon_{z}\right)_{D}$ and $\left(\gamma_{y z}\right)_{D}$ due to distortion are not zero. However, as mentioned in assumption $\mathrm{f}$ ), if the displacement in the direction of coordinate $n$ due to lateral bending of the plate is assumed to be zero, and only the member-rotation as a rigid body is considered, the following equations would be obtained, also taking into account assumption d) :

$\left(\varepsilon_{y}\right)_{D}=0, \quad\left(\varepsilon_{z}\right)_{D}=0, \quad\left(\gamma_{y z}\right)_{D}=0$.

b) Condition of continuous warping

As shown in Eqs. (8), the warping $u_{1}$ of a straight beam corresponds to $\left(R_{N} / \rho\right) u_{1}$ of a curved beam. Therefore, the condition of continuous warping can be expressed by the following equation.

$$
\oint_{(i)} \frac{\partial}{\partial s}\left(\frac{R_{N}}{\rho} u_{1}\right) d s=0 \quad(i=1,2, \cdots, m)
$$

In this Eq. (10), $i$ is the cell number and $m$ is the total number of cells. This equation can be conveniently divided into the two followings using Eq. (6), so that it conforms with the theory of torsion bending :

$$
\oint_{(i)} \frac{\partial}{\partial s}\left(\frac{R_{N}}{\rho} u\right) d s=0, \quad \oint_{(i)} \frac{\partial}{\partial s}\left(\frac{R_{N}}{\rho} u_{2}\right) d s=0 \quad(i=1,2, \cdots, m)
$$

By substituting Eq. $(9 \cdot \mathrm{b})$ into Eq. (IV), the following equation is obtained :

$$
\oint_{(i)} \frac{R_{N}}{\rho} \gamma_{x s, s e} d s=0 \quad(i=1,2, \cdots, m)
$$

c) Equilibrium condition of forces acting on an infinitesimal plate element

If the length of two sides of plate element with thickness $t$ can be given as $\rho \cdot d \theta$ and $d s$, the follwing relationship exists between normal stress $\sigma_{\theta}$, shearing stress $\tau_{s \theta}$ and distributed load $p_{N}(\theta, s)^{14)}$ :

$$
\frac{1}{\rho^{2}} \frac{\partial}{\partial s}\left(\rho^{2} \tau_{s \theta} t\right)+\frac{1}{\rho} \frac{\partial}{\partial \theta}\left(\sigma_{\theta} t\right)+p_{N} t=0
$$

In order to change the differential $\theta$ above to $x,\left(R_{N} / \rho\right) \sigma_{x}$ is used instead of $\sigma_{\theta}$, in a similar manner to the conversion of $u_{1}$, and Eq. (3) is substituted into Eq. (11). The following equation is obtained :

$$
\left(\frac{R_{N}}{\rho}\right)^{2} \frac{\partial}{\partial s}\left[\left(\frac{\rho}{R_{N}}\right)^{2} \tau_{x s} t\right]+\left(\frac{R_{N}}{\rho}\right)^{2} \frac{\partial}{\partial x}\left(\sigma_{x} t\right)+p_{N} t=0
$$

As the primary shearing stress does not relate to the normal stress, the Eq. (12) can be divided into the two following equations :

$$
\left(\frac{R_{N}}{\rho}\right)^{2} \frac{\partial}{\partial s}\left[\left(\frac{\rho}{R_{N}}\right)^{2} \tau_{x s, p r} t\right]=0, \quad\left(\frac{R_{N}}{\rho}\right)^{2} \frac{\partial}{\partial s}\left[\left(\frac{\rho}{R_{N}}\right)^{2} \tau_{x s, s e} t\right]+\left(\frac{R_{N}}{\rho}\right)^{2} \frac{\partial}{\partial x}\left(\sigma_{x} t\right)+p_{N} t=0
$$

\section{(3) Displacement in the plane of cross-section}

In Eqs. (8), as the definitions of strain components $\varepsilon_{y}, \varepsilon_{z}$ and $\gamma_{y z}$ are identical to those of a straight 
beam, the displacements in the plane of cross-section can be derived from a simple integral calculus using strain conditions (I) and (II), as in the case of straight beam, as follows ${ }^{11)}$ :

$$
\left.\begin{array}{l}
v=\eta(x)-\left(z-z_{M}\right) \varphi(x)-\sum_{i=1}^{m}\left(z-z_{v i}\right) a_{i}(s) \gamma_{i}(x) \\
w=\zeta(x)+\left(y-y_{M}\right) \varphi(x)+\sum_{i=1}^{m}\left(y-y_{v i}\right) a_{i}(s) \gamma_{i}(x)
\end{array}\right\}
$$

Where, $\eta(x)$ and $\zeta(x)$ are the bending displacements in the direction of axes $y$ and $z$, respectively, $\varphi(x)$ is the angle of rotation, $\gamma_{i}(x)$ represents the magnitude of the basic distortion (i). The coordinates of shear center $M$ are $y_{M}, z_{M}$, and the coordinates of the center of rotation of each plate at the basic distortion (i ) are $y_{v i}, z_{v i}$. A function $a_{i}(s)$, which takes a constant value for each plate, gives a linear correlation between the angle of rotation of each plate for the basic distortion ( $i)$ and the distortional angle $\gamma_{i}(x)$. From the above equations, the displacement $f$ becomes as follows :

$$
f=\eta(x) \cos \alpha+\zeta(x) \sin \alpha+\varphi(x) r_{T}+\sum_{i=1}^{m} \gamma_{i}(x) r_{P i}
$$

Where, $\alpha$ is the angle between the $y$ axis and each plate. The distance from the shear center to each plate $r_{T}$ and that from the distortional center of rotation of each plate to the plate $r_{P i}$ are expressed as follows :

$$
\left.\begin{array}{l}
r_{T}=\left(y-y_{M}\right) \sin \alpha-\left(z-z_{M}\right) \cos \alpha \\
r_{P_{i}}=\left[\left(y-y_{v i}\right) \sin \alpha-\left(z-z_{v i}\right) \cos \alpha\right] a_{i}(s) \quad(i=1,2, \cdots, m)
\end{array}\right\}
$$

(4) Displacements in the directions of the coordinates $x$ and $\rho$

By integrating Eq. (V) and applying Hooke's Law, the following equations can be obtained.

$$
\gamma_{x s, p r}=\frac{1}{G}\left(\frac{R_{N}}{\rho}\right)^{2} \frac{T(x)}{t}, \quad \tau_{x s, p r}=\left(\frac{R_{N}}{\rho}\right)^{2} \frac{T(x)}{t} \text {. }
$$

Where, $G$ is the shear modulus, $T(x)$ is an integral constant arising from the integration with respect to $s$. By substituting this Eq. (16 - a) into Eq. $(9 \cdot a)$ and integrating with respect to $s$, the warping can be expressed as below using the condition of continuous warping (III) :

$$
\begin{aligned}
u(x, s)= & -\left[1+\left(\frac{\cos \beta}{R_{N}}\right) y-\left(\frac{\sin \beta}{R_{N}}\right) z\right] \xi^{\prime}(x)-\left[\frac{R_{N}}{R_{M}}\left(1+\frac{y_{M}}{R_{N}} \cos \beta\right) y-\left(\frac{y_{M}}{R_{M}} \sin \beta\right) z-\left(\frac{\sin \beta}{R_{M}}\right) w_{T}\right] \eta^{\prime}(x) \\
& -\left[\left(\frac{z_{M}}{R_{M}} \cos \beta\right) y+\frac{R_{N}}{R_{M}}\left(1-\frac{z_{M}}{R_{N}} \sin \beta\right) z-\left(\frac{\cos \beta}{R_{M}}\right) w_{T}\right] \zeta^{\prime}(x)-w_{T} \varphi^{\prime}(x)-\sum_{i=1}^{m} w_{P i} \gamma_{i}^{\prime}(x) \cdots \cdots(17)
\end{aligned}
$$

The unit warping functions can be defined as follows :

$$
\left.\begin{array}{l}
y(s)=\int_{0}^{s} \cos \alpha d s, \quad z(s)=\int_{0}^{s} \sin \alpha d s, \quad w_{T}(s)=\frac{\rho(s)}{R_{N}} \int_{0}^{s}\left\{\left(\frac{R_{N}}{\rho(s)}\right)^{2} r_{T}-\left(\frac{R_{N}}{\rho(s)}\right)^{3} \frac{\Psi_{T}}{t}\right\} d s \\
w_{P i}(s)=\frac{\rho(s)}{R_{N}} \int_{0}^{s}\left\{\left(\frac{R_{N}}{\rho(s)}\right)^{2} r_{P i}-\left(\frac{R_{N}}{\rho(s)}\right)^{3} \frac{\Psi_{P i}}{t}\right\} d s \quad(i=1,2, \cdots, m)
\end{array}\right\}
$$

Where, $-\xi^{\prime}(x)$ is an integral constant arising from the integration with respect to $s$ and geometrically equals the magnitude of displacement of the point $\mathrm{N}$ in the direction of the $x$ axis. The definition of the primary shear flow function of torsion $\Psi_{T}$ will be omitted here as that has been given in various references. The primary shear flow function of distortion $\Psi_{P i}$ can be expressed in the same form as that of torsion. $R_{M}$ is the distance between the center of curvature and the shear center.

Displacement in the direction of the coordinate $\rho$ at an arbitrary point $\mathrm{A}$ on the coordinate $s$ of the cross-section can be given by rearranging Eqs. (13), as below :

$$
\begin{aligned}
r= & -R_{N}\left\{\left[-\frac{\cos \beta}{R_{N}} \eta(x)+\frac{\sin \beta}{R_{N}} \zeta(x)-\left(\frac{y_{M}}{R_{N}} \sin \beta+\frac{z_{M}}{R_{N}} \cos \beta\right) \varphi(x)\right] 1\right. \\
& \left.+\left[\frac{\sin \beta}{R_{N}} \varphi(x)\right] y+\left[\frac{\cos \beta}{R_{N}} \varphi(x)\right] z\right\} \ldots \ldots \ldots \ldots \ldots \ldots \ldots \ldots \ldots \ldots \ldots \ldots \ldots \ldots \ldots \ldots \ldots \ldots \ldots \ldots \ldots \ldots \ldots
\end{aligned}
$$

However, the displacement component due to distortion is disregarded as it is extremely small compared with that due to bending, as stated in assumption $h$ ). 


\section{( 5 ) Stress}

To simplify the derivation of the equations, a vector representation is introduced. The displacement function vector $\boldsymbol{V}$, direction vector $\boldsymbol{r}$, primary shear flow vector $\boldsymbol{Y}$ and unit warping function vector $\boldsymbol{w}$ are defined below (Superscript $T$ means transpose) :

$$
\begin{aligned}
& \boldsymbol{V}^{T}=\left(\begin{array}{lllllll}
\xi & \eta & \zeta & \varphi & \gamma_{1} & \cdots & \gamma_{m}
\end{array}\right), \quad \boldsymbol{r}^{T}=\left(\begin{array}{llllllll}
0 & \cos \alpha & \sin \alpha & r_{T} & r_{P 1} & \cdots & r_{P m}
\end{array}\right) \\
& \boldsymbol{\Psi}^{T}=\left(\begin{array}{llllllllll}
0 & 0 & 0 & \Psi_{T} & \Psi_{P 1} & \cdots & \Psi_{P m}
\end{array}\right), \quad \boldsymbol{w}^{T}=\left(\begin{array}{lllllll}
1 & y & z & w_{T} & w_{P 1} & \cdots & w_{P m}
\end{array}\right)
\end{aligned}
$$

Using these equations Eqs. (17) and (19) can be rewritten as below :

$$
u(x, s)=-\boldsymbol{w}^{T} \boldsymbol{B} \boldsymbol{V}^{\prime}, \quad r(x, s)=-R_{N} \boldsymbol{w}^{T} \boldsymbol{C V}
$$

Where, matrices $B$ and $C$ become as shown below:
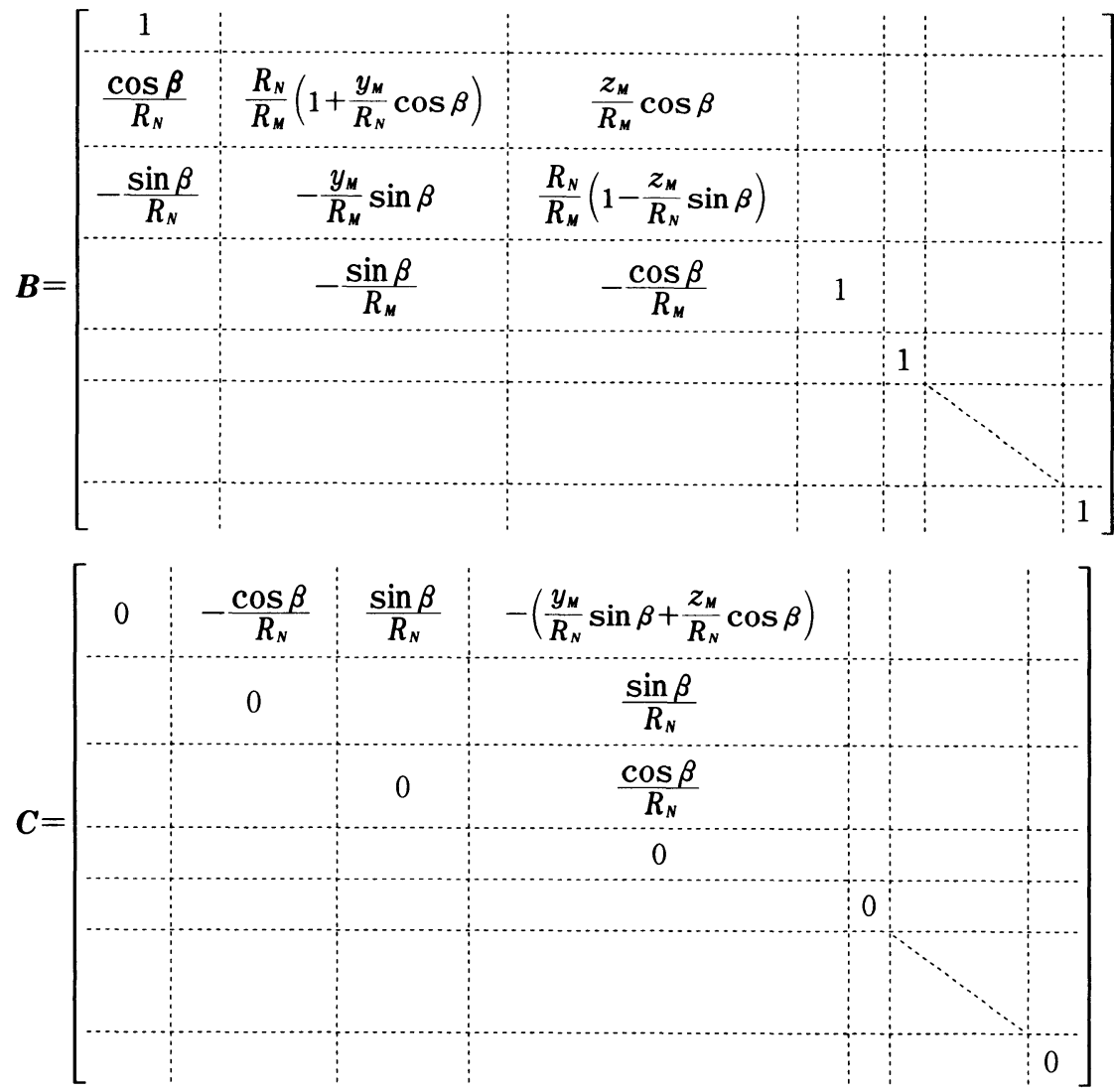

Blank sections in these matrices are all zero elements.

Assuming that the axial displacements $u_{1}$ and $u_{2}$ hold the unit warping function in common, Eq. (21 - a) can be divided into the equations shown below using the relationship in Eq. (6) :

$$
u_{1}(x, s)=-\boldsymbol{w}^{T} \boldsymbol{B} \boldsymbol{V}_{1}^{\prime}, \quad u_{2}(x, s)=-\boldsymbol{w}^{T} \boldsymbol{B} \boldsymbol{V}_{2}^{\prime}
$$

However, the following relationship exists :

$$
V_{1}=V-V_{2}
$$

By substituting Eqs. $(21 \cdot \mathrm{b})$ and $(22 \cdot \mathrm{a})$ into Eq. $(8 \cdot \mathrm{a})$ and applying Hooke's Law, the following equations can be obtained :

$$
\varepsilon_{x}=-\frac{R_{N}}{\rho} \boldsymbol{w}^{T}\left(\boldsymbol{B} \boldsymbol{V}_{1}^{\prime \prime}+\boldsymbol{C V}\right), \quad \sigma_{x}=-E \frac{R_{N}}{\rho} \boldsymbol{w}^{T}\left(\boldsymbol{B} \boldsymbol{V}_{1}^{\prime \prime}+C \boldsymbol{V}\right)
$$

Where, $E$ is the Young's modulus.

The primary shearing strain and stress can be expressed using the condition of continuous warping (III) 
by the following equations :

$$
\gamma_{x s, \rho r}=\left(\frac{R_{N}}{\rho}\right)^{2} \frac{\Psi^{T}}{t} B V^{\prime}, \quad \tau_{x s, \rho r}=G\left(\frac{R_{N}}{\rho}\right)^{2} \frac{\Psi^{T}}{t} B V^{\prime}
$$

The secondary shearing strain and stress can be obtained using either of the two methods. That is, by substituting Eq. $(22 \cdot \mathrm{b})$ into Eq. $(9 \cdot \mathrm{b})$, and using Hooke's Law, the following equations are obtained :

$$
\frac{R_{N}}{\rho} \tilde{\gamma}_{x s, s e}=\frac{\partial}{\partial s}\left(\frac{R_{N}}{\rho} \boldsymbol{w}^{T}\right) B V_{2}^{\prime}, \quad \frac{R_{N}}{\rho} \tilde{\tau}_{x s, s e}=G \frac{\partial}{\partial s}\left(\frac{R_{N}}{\rho} \boldsymbol{w}^{T}\right) B V_{2}^{\prime}
$$

Symbol is supplemented to distinguish them from the shearing strain and stress to be obtained by another method. The other method is a method using the equilibrium condition of forces (V). By substituting Eq. $(24 \cdot \mathrm{b})$ into Eq. (V) and carrying out integral calculus with respect to $s$ and applying Hooke's Law and determining the constant of integration from Eq. (IV)', the following equations are obtained :

$$
\begin{aligned}
& \gamma_{x s, s e}=\frac{E}{G}\left(\frac{R_{N}}{\rho}\right)^{2} \frac{(\boldsymbol{S}-\Phi)^{T}}{t}\left(B \boldsymbol{V}_{1}^{\prime \prime \prime}+\boldsymbol{C} \boldsymbol{V}^{\prime}\right)-\frac{1}{G}\left(\frac{R_{N}}{\rho}\right)^{2} \frac{f_{N}-q_{N}}{t} \\
& \tau_{x s, s e}=E\left(\frac{R_{N}}{\rho}\right)^{2} \frac{(\boldsymbol{S}-\boldsymbol{\Phi})^{T}}{t}\left(\boldsymbol{B} \boldsymbol{V}_{1}^{\prime \prime \prime}+\boldsymbol{C} \boldsymbol{V}^{\prime}\right)-\left(\frac{R_{N}}{\rho}\right)^{2} \frac{f_{N}-q_{N}}{t}
\end{aligned}
$$

Where, the follwing definitions shall apply:

$$
S(s)=\int_{s_{0}}^{s} \frac{R_{N}}{\rho(s)} \boldsymbol{w} d F, \quad f_{N}(x, s)=\int_{s_{0}}^{s}\left(\frac{\rho(s)}{R_{N}}\right)^{2} p_{N} d F
$$

Vector $\Phi$ and function $q_{N}$ take constant values for each cell of the cross-section (see Ref.4)).

From the above the total shearing strain and stress can be shown as the sum of Eqs. (25) and (27), as follows :

$$
\begin{aligned}
& \gamma_{x s}=\left(\frac{R_{N}}{\rho}\right)^{2} \frac{\boldsymbol{\Phi}^{T}}{t} \boldsymbol{B} \boldsymbol{V}^{\prime}+\frac{E}{G}\left(\frac{R_{N}}{\rho}\right)^{2} \frac{(\boldsymbol{S}-\boldsymbol{\Phi})^{T}}{t}\left(\boldsymbol{B} \boldsymbol{V}_{1}^{\prime \prime \prime}+\boldsymbol{C} \boldsymbol{V}^{\prime}\right)-\frac{1}{G}\left(\frac{R_{N}}{\rho}\right)^{2} \frac{f_{N}-q_{N}}{t} \\
& \tau_{x s}=G\left(\frac{R_{N}}{\rho}\right)^{2} \frac{\boldsymbol{\Phi}^{T}}{t} \boldsymbol{B} \boldsymbol{V}^{\prime}+E\left(\frac{R_{N}}{\rho}\right)^{2} \frac{(\boldsymbol{S}-\boldsymbol{\Phi})^{T}}{t}\left(\boldsymbol{B} \boldsymbol{V}_{1}^{\prime \prime \prime}+\boldsymbol{C} \boldsymbol{V}^{\prime}\right)-\left(\frac{R_{N}}{\rho}\right)^{2} \frac{f_{N}-q_{N}}{t}
\end{aligned}
$$

In the secondary shearing strain and stress obtained by the two methods, if the equivalence of the work done by them is assumed, the following equations of vitrual force and virtual displacement would be given.

$$
\int_{V} \delta \tau_{x s, s e} \gamma_{x s, s e} d V=\int_{V} \delta \tau_{x s, s e} \tilde{\gamma}_{x s, s e} d V, \quad \int_{V} \tau_{x s, s e} \delta \gamma_{x s, s e} d V=\int_{V} \tau_{x s, s e} \delta \tilde{\gamma}_{x s, s e} d V
$$

Where, $\delta$ means the virtual component and $\int_{V} \cdots d V$ means the volume integral. By substituting Eqs. (26) and (27) in this Eq. $(30 \cdot$ a) and by rearranging, the following equation is obtained :

$$
\frac{E}{G} \boldsymbol{R}\left(\boldsymbol{B} \boldsymbol{V}_{1}^{\prime \prime \prime}+\boldsymbol{C} \boldsymbol{V}^{\prime}\right)-\frac{1}{G} \int_{F}\left(\frac{R_{N}}{\rho}\right)^{3} \frac{(\boldsymbol{S}-\boldsymbol{\Phi})}{t} \frac{\left(f_{N}-q_{N}\right)}{t} d F+\boldsymbol{F} \boldsymbol{B}\left(\boldsymbol{V}^{\prime}-\boldsymbol{V}_{1}^{\prime}\right)=\mathbf{0}
$$

where warping resistance matrix $\boldsymbol{F}$ and shear correction matrix $\boldsymbol{R}$ are defined by

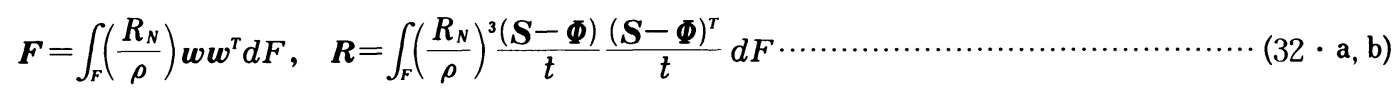

\section{(6) Orthogonal conditions}

By premultiplying the equation obtained by substituting Eq. (21 - a) into Eq. (III) and the equation obtained by substituting Eq. (27-a) into Eq. (IN)', by constant valued vector respectively, the following orthogonal conditions can be obtained :

$$
\left.\begin{array}{l}
\int_{F} \frac{\boldsymbol{T}}{t} \frac{\partial}{\partial s}\left(\frac{R_{N}}{\rho} \boldsymbol{w}^{T}\right) d F=0 \\
\frac{E}{G} \int_{F}\left(\frac{R_{N}}{\rho}\right)^{3} \frac{\boldsymbol{T}}{t} \frac{(\boldsymbol{S}-\boldsymbol{\Phi})^{T}}{t} d F\left(\boldsymbol{B} \boldsymbol{V}_{1}^{\prime \prime \prime}+\boldsymbol{C} \boldsymbol{V}^{\prime}\right)-\frac{1}{G} \int_{F}\left(\frac{R_{N}}{\rho}\right)^{3} \frac{\boldsymbol{\Psi}}{t} \frac{\left(f_{N}-q_{N}\right)}{t} d F=0
\end{array}\right\}
$$

\section{( 7 ) Lateral bending moment}

If the element of curved girder in which the length of the neutral axis is 1 is cut out, assuming this to be a box frame, the following flexibility relation exists between vector $m$ of the unknown lateral bending moment at each corner and vector $\gamma$ of the change in the angle of the corner : 
$\Delta \boldsymbol{m}-E \boldsymbol{\gamma}=\mathbf{0}$

The flexibility matrix $\Delta$ is given as shown by the following equations :

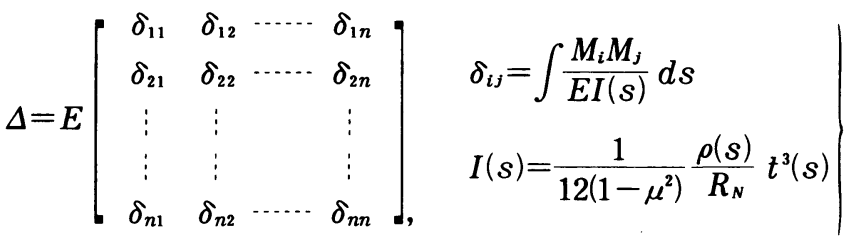

Where, $M_{i}$ is a function of bending moment when a couple of moments in the opposite direction of magnitude 1 is acting on the corner $\langle i\rangle$ in a "primary structural system" and $\mu$ is the Poission's ratio. The vector of the change in angle of the corner can be made to correspond with the displacement function vector $V$ using transformation matrix $\boldsymbol{T}$, which calculate the difference in the angle of rotation between each plate and the adjacent plate for the basic distortion, as follows ${ }^{15}$ :

$\gamma=\boldsymbol{T V}$

By eliminating $\gamma$ from Eqs. (34) and (36) the lateral bending moment vector $\boldsymbol{m}$ is expressed as follows :

$$
\boldsymbol{m}=E \Delta^{-1} \boldsymbol{T V} \text {. }
$$

(8) Governing differential equation

By expressing the virtual work of an external forces as $A_{a}$ and that of an internal forces as $A_{i}$, from the principle of virtual displacement the following can be obtained :

$$
\begin{aligned}
& \delta A_{a}+\delta A_{i}=0 \\
& \delta A_{a}=\int_{\theta} \int_{F} p_{Q} \delta v_{Q} d s \rho d \theta+\int_{\theta} \int_{F} p_{N} \delta u_{1} d F \rho d \theta+\sum_{i} P_{Q i} \delta v_{Q i}+\sum_{j} P_{N j} \delta u_{1 j} \\
& \delta A_{i}=-\int_{\theta} \int_{F}\left(\sigma_{x} \delta \varepsilon_{x}+\sigma_{s} \delta \varepsilon_{s}+\sigma_{n} \delta \varepsilon_{n}+\tau_{x s} \delta \gamma_{x s}+\tau_{s n} \delta \gamma_{s n}+\tau_{n x} \delta \gamma_{n x}\right) d F \rho d \theta
\end{aligned}
$$

Where $p_{Q}$ and $P_{Q}$ are the distributed load and concentrated load acting transverse to the axis of the beam, and $p_{N}$ and $P_{N}$ are the distributed load and concentrated load acting parallel to the axis of the beam.

From assumption b) $\sigma_{n}=\tau_{s n}=\tau_{n x} \fallingdotseq 0$ in the above equation. Also, the virtual work of normal stress $\sigma_{s}$ due to cross-sectional distortion can be rewritten as the virtual work of lateral bending moment. Therefore, by replacing the variable using $\mathrm{Eq}$. (3), the following equations can be derived.

$$
\begin{aligned}
\delta A_{a} & =\int_{l} \int_{F} \frac{\rho}{R_{N}} p_{Q} \delta v_{Q} d s d x+\int_{l} \int_{F} \frac{\rho}{R_{N}} p_{N} \delta u_{1} d F d x+\sum_{i} P_{Q i} \delta v_{Q i}+\sum_{j} P_{N j} \delta u_{1 j} \\
\delta A_{i} & =-\int_{l}\left[\int_{F} \frac{\rho}{R_{N}}\left(\sigma_{x} \delta \varepsilon_{x}+\tau_{x s} \delta \gamma_{x s}\right) d F+\boldsymbol{m}^{T} \delta \gamma\right] d x
\end{aligned}
$$

Displacement $v_{Q}$ in the direction of transverse load action $p_{Q}$ or $P_{Q}$ can be given as below :

$$
v_{Q}(x, s)=\boldsymbol{r}^{* T} \boldsymbol{V}
$$

Where the vector $r^{*}$ is defined as follows :

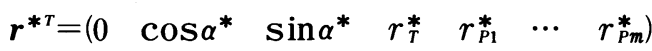

in which $\alpha^{*}$ is the angle between the $y$-axis and the direction of the load acton, $r_{T}^{*}$ is the distance from the shear center $\mathrm{M}$ to line of action of the load and $r_{P i}^{*}$ is the distance from the center of rotation $\mathrm{V}$ to line of action of the load when the basic cross-sectional distortion (i) occurs (see Ref. 16)) .

By substituting Eq. (40) into Eq. (38), and also substituting Eqs. (22 - a), (24) and (29) and by rearranging them using the relationship in $\mathrm{Eq} .(30 \cdot \mathrm{b})$, finally the following governing equation and two boundary conditions can be obtained :

$$
\begin{aligned}
& E \boldsymbol{B}^{T} \boldsymbol{F}\left(\boldsymbol{B} \boldsymbol{V}_{1}^{1 v}+C \boldsymbol{V}^{\prime \prime}\right)-G \boldsymbol{B}^{T} \boldsymbol{J} \boldsymbol{B} \boldsymbol{V}^{\prime \prime}+E \boldsymbol{C}^{T} \boldsymbol{F}\left(\boldsymbol{B} \boldsymbol{V}_{1}^{\prime \prime}+\boldsymbol{C} \boldsymbol{V}\right)+E \boldsymbol{I}_{K} \boldsymbol{V}=\int_{F} \frac{\rho}{R_{N}} p_{Q} r^{*} d s+\boldsymbol{B}^{T} \int_{F} \frac{\rho}{R_{N}} p_{N}^{\prime} \boldsymbol{w} d F
\end{aligned}
$$

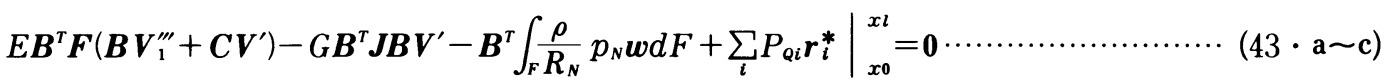

$$
\begin{aligned}
& E \boldsymbol{B}^{T} \boldsymbol{F}\left(\boldsymbol{B} \boldsymbol{V}_{1}^{\prime \prime}+\boldsymbol{C V}\right)+\left.\boldsymbol{B}^{T} \sum_{j} P_{N j} \boldsymbol{w}_{j}\right|_{x_{0}} ^{x l}=0
\end{aligned}
$$


Where, torsional resistance matrix $J$ and frame resistance matrix $I_{K}$ are defined as follows :

$$
J=\int_{F}\left(\frac{R_{N}}{\rho}\right)^{3} \frac{\boldsymbol{T}}{t} \frac{\boldsymbol{T}^{T}}{t} d F, \quad I_{K}=T^{T} \Delta^{-1} \boldsymbol{T}
$$

(9) Stress resultants

The warping moment vector $\boldsymbol{M}$, primary shearing force vector $\boldsymbol{Q}_{p r}$, secondary shearing force vector $\boldsymbol{Q}_{s e}$ and total shearing force vector $\boldsymbol{Q}$ are defined as follows :

$$
\boldsymbol{M}=\int_{F} \sigma_{x} w d F, \quad Q_{p r}=\int_{F} \tau_{x s, p r} r d F, \quad Q_{s e}=\int_{F} \tau_{x s, s e} r d F, \quad \boldsymbol{Q}=\int_{F} \tau_{x s} r d F \cdots \cdots \cdots \cdots \cdots \cdots \cdots \cdots \cdots \cdots \cdots \cdots \cdots(45 \cdot \mathrm{a} \sim \mathrm{d})
$$

These elements are expressed specifically as follows:

$$
\begin{aligned}
& M^{T}=\left(\begin{array}{lllllll}
N_{x} & M_{z} & M_{y} & M_{w T} & M_{w P 1} & \cdots & M_{w P m}
\end{array}\right), \quad Q_{p r}^{T}=\left(\begin{array}{lllllll}
0 & 0 & 0 & M_{T, p r} & Q_{P 1, p r} & \cdots & Q_{P m, p r}
\end{array}\right) \\
& \boldsymbol{Q}_{s e}^{T}=\left(\begin{array}{lllllllllllll}
0 & Q_{y} & Q_{z} & M_{T, s e} & Q_{P 1, s e} & \cdots & Q_{P m, s e}
\end{array}\right), \quad Q^{T}=\left(\begin{array}{lllllll}
0 & Q_{y} & Q_{z} & M_{T} & Q_{P 1} & \cdots & Q_{P m}
\end{array}\right.
\end{aligned}
$$

However, following relationships exist :

$$
M_{T}=M_{T, p r}+M_{T, s e}, \quad Q_{P 1}=Q_{P 1, p r}+Q_{P 1, s e}, \cdots \cdots, \quad Q_{P m}=Q_{P m, p r}+Q_{P m, s e}
$$

By substituting Eqs. $(24 \cdot$ b) , $(25 \cdot b),(27 \cdot b)$ and $(29 \cdot b)$ into the stress terms of Eqs. (45), and by rearranging, the following equations can be obtained :

$$
\begin{aligned}
& M=-E F\left(B V_{1}^{\prime \prime}+C V\right), \quad Q_{p r}=G B^{T} J B V^{\prime}, \quad Q_{s e}=-E B^{T} F\left(B V_{1}^{\prime \prime \prime}+C V^{\prime}\right)+B^{T} \int_{F} \frac{\rho}{R_{N}} p_{N} w d F
\end{aligned}
$$

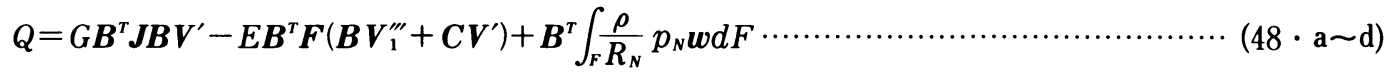

From these equations the following expression can be obtained :

$$
Q_{s e}=B^{r}\left(M^{\prime}+\int_{F} \frac{\rho}{R_{N}} p_{N} w d F\right)
$$

(10) Orthogonalization of warping resistance matrix

When the principal coordinate system in Fig. 1 is adopted, all the unit warping functions based on the assumption of rigid cross-section form orthogonal set with one another. However, as the basic distortions are optionally selected, their unit warping functions are not orthogonal. These unit warping functions of distortion are orthogonalized successively under the three following conditions :

i ) "Warpings of distortion" and "warpings of rigid cross-section" are orthogonal. ii) "Primary shearing strain of distortion" and "primary shearing strain of torsion" are orthogonal. iii) "Warpings of distortion" are orthogonal with one another and at the same time "primary shearing strains of distortion" are orthogonal.

From the above three conditions a matrix of orthogonalization $K$ is obtained and by using it, transformation of the matrices of various sectional properties can be performed (see Ref. 11)). Matrices $B$ and $C$, which are charecteristic of curved beams, can be transformed as follows :

$K^{-1} B K \Rightarrow B, K^{-1} C K \Rightarrow C$

(11) Relationships between the stress and stress resultant

As vector $V^{\prime}$ is eliminated from Eqs. (25 - b) and (48 b), which represent primary shearing stress by means of the stress resultant, the following equation is obtained :

$$
\tau_{x s, p r}=\left(\frac{R_{N}}{\rho}\right)^{2} \frac{\tau^{* T}}{t} J^{*-1} Q_{p r}^{*}
$$

Where, $J^{*}$ is a nonsingular matrix obtained by deleting the row and column of the terms containing zero diagonal elements in matrix $J . \mathbf{T}^{*}$ and $\boldsymbol{Q}_{p r}^{*}$ are vectors that can be obtained by deleting the elements corresponding to the transformation from $J$ to $J^{*}$.

Similarly, by eliminating $\left(\boldsymbol{B} \boldsymbol{V}_{1}^{\prime \prime}+\boldsymbol{C V}\right)$ from Eqs. $(24 \cdot \mathrm{b})$ and $(48 \cdot \mathrm{a})$ and $\left(\boldsymbol{B} \boldsymbol{V}_{1}^{\prime \prime \prime}+\boldsymbol{C} \boldsymbol{V}^{\prime}\right)$ from Eqs. $(27 \cdot b)$ and $(48 \cdot c)$, the stresses can be represented by means of the stress resultants, as follows :

$$
\sigma_{x}=\frac{R_{N}}{\rho} \boldsymbol{w}^{T} \boldsymbol{F}^{-1} \boldsymbol{M}, \quad \tau_{x s, s e}=-\left(\frac{R_{N}}{\rho}\right)^{2} \frac{(\boldsymbol{S}-\boldsymbol{\Phi})^{T}}{. t} \boldsymbol{F}^{-1}\left(\boldsymbol{B}^{T}\right)^{-1} \boldsymbol{Q}_{s e}
$$


However, the load terms are omitted in this description.

(12) System of first-order ordinary differential equations

The derivative of the displacement function vector $V_{1}$ is called here the "generalized slope vector" : $\theta_{1}=V_{1}^{\prime}$

and the state vector $z$ is defined as follows :

$\boldsymbol{z}^{T}=\left(\begin{array}{l:l:l:l}\boldsymbol{V} & \boldsymbol{\theta}_{1} & \boldsymbol{M} & \boldsymbol{Q}\end{array}\right)$

As a system of ordinary differential equations in $z$ is obtained using Eqs. (43 - a), (48) and (53),

$\frac{d z}{d x}=\boldsymbol{A z}+\boldsymbol{b}(\boldsymbol{x})$

Where, coefficient matrix $\boldsymbol{A}$ and load vector $\boldsymbol{b}(x)$ are defined by Eqs. (57) and (58), respectively, in which $E$ is a unit matrix and $D$ is defined by the following equation :

$\boldsymbol{D}=\boldsymbol{F}^{-1} \boldsymbol{R} \boldsymbol{F}^{-1}$

$$
\begin{aligned}
& \boldsymbol{A}=\left[\begin{array}{c:c:c:c}
0 & \boldsymbol{B}^{-1}(\boldsymbol{E}+\boldsymbol{D J})^{-1} \boldsymbol{B} & 0 & \frac{1}{G} \boldsymbol{B}^{-1}(\boldsymbol{E}+\boldsymbol{D} \boldsymbol{J})^{-1} \boldsymbol{D}\left(\boldsymbol{B}^{T}\right)^{-1} \\
\hdashline-\boldsymbol{B}^{-1} \boldsymbol{C} & 0 & -\frac{1}{E} \boldsymbol{B}^{-1} \boldsymbol{F}^{-1} & 0 \\
\hdashline 0 & -G \boldsymbol{J}(\boldsymbol{E}+\boldsymbol{D} \boldsymbol{J})^{-1} \boldsymbol{B} & \mathbf{0} & {\left[\boldsymbol{E}-\boldsymbol{J}(\boldsymbol{E}+\boldsymbol{D J})^{-1} \boldsymbol{D}\right]\left(\boldsymbol{B}^{T}\right)^{-1}} \\
\hdashline \boldsymbol{E} \boldsymbol{I}_{\boldsymbol{K}} & \mathbf{0} & -\boldsymbol{C}^{T} & 0
\end{array}\right] \\
& \boldsymbol{b}(x)=\left[\begin{array}{c}
\frac{1}{G} \boldsymbol{B}^{-1}(\boldsymbol{E}+\boldsymbol{D} \boldsymbol{J})^{-1}\left\{\boldsymbol{F}^{-1} \int_{F}\left(\frac{R_{N}}{\rho}\right)^{3} \frac{(\boldsymbol{S}-\boldsymbol{\Phi})}{t} \frac{\left(f_{N}-q_{N}\right)}{t} d F-\boldsymbol{D} \int_{F} \frac{\rho}{R_{N}} p_{N} \boldsymbol{w} d F\right\} \\
\mathbf{0} \\
-\boldsymbol{J}(\boldsymbol{E}+\boldsymbol{D} \boldsymbol{J})^{-1} \boldsymbol{F}^{-1} \int_{F}\left(\frac{R_{N}}{\rho}\right)^{3} \frac{(\boldsymbol{S}-\boldsymbol{\Phi})}{t} \frac{\left(f_{N}-q_{N}\right)}{t} d F-\left\{\boldsymbol{E}-\boldsymbol{J}(\boldsymbol{E}+\boldsymbol{D J})^{-1} \boldsymbol{D}\right\} \int_{F} \frac{\rho}{R_{N}} p_{N} \boldsymbol{w} d F \\
\cdots \\
\cdots \int_{F} \frac{\rho}{R_{N}} p_{Q} \boldsymbol{r}^{*} d s
\end{array}\right]
\end{aligned}
$$

\section{CONCLUSION}

Governing equations of curved, multi-cell box girder bridges with deformable cross-section were derived, in which the effects of shearing strain and variation of longitudinal fibre curvature were taken into account. Therefore this theory as it is developed here can deal with continuous, curved girder with large curvature.

In the theory, it was shown that displacement $u$ of a straight girder corresponds to $\left(R_{N} / \rho\right) u$ of a curved girder, and a change was made to the equilibrium condition of the forces. Due to this change the equilibrium of forces is not satisfied in a strict sense, but this was considered to be unavoidable in order to maintain conventional theory and to secure an uniqueness of distribution of secondary shearing stress.

While deriving the governing equations, coefficient matrices $B$ and $\boldsymbol{C}$ were introduced, aiming at correspondence with the case of a straight girder. As $R_{N} / \rho$ equals 1 in the case of a straight girder, matrices $B$ and $C$ are transformed to a unit matrix and a zero matrix, respectively. Transformed the equations of this report, such as this, the equations become the equations of straight, multi-cell box girder (see Ref. ${ }^{11)}$ ).

\section{REFERECES}

1) Konishi, I. and Komatsu, S. : On fundamental theory of thin-walled curved girder, Proc. of JSCE, No. 87, pp. 35 48, Nov. , 1962 (in Japanese).

2) Kuranishi, S. : Anaysis of thin-walled cerved beams, Proc. of JSCE, No. 108, pp. 7 12, Aug., 1964 (in Japanese) 
3) Fukasawa, Y. : Fundamental theory on the statical analysis of thin-walled curved bars, Proc. of JSCE, No. 110, pp. 30 51, Oct., 1964 (in Japanese).

4) Nishino, F. and Fukasawa, Y. : Formulation of static behavior of thin-walled curved beams under assumption of strain field, Proc. of JSCE, No.247, pp.9 19, March, 1976 (in Japanese).

5) Kambe, S. : Modified theory of torsion bending for a circularly curved box girder bridges, Proc. of JSCE, No. 297, pp. 95 110, May, 1980.

6) Otsuka, H., Yoshimura, T. and Hikosaka, H. : Stiffening effects of intermediate diaphragms in curved box girder bridges, Bridge and Foundation Engineering, Vol. 12, No. 1, pp. 39 42, No. 2, pp. 40 43, 1978 (in Japanese).

7) Sakai, F. and Nagai, M. : Three-dimensional analysis of thin-walled curved box girders by block finite element method, Proc. of JSCE, No. 295, pp. 1 12, March, 1980 (in Japanese).

8) Dabrowski, R. : Gekrümmte dünnwandige Träger, Springer-Verlag, 1968.

9) Nakai, H. and Murayama, Y. : Distortional stress analysis and design aid for horizontally curved box girder bridges with diaphragms, Proc. of JSCE, No. 309, pp. 25 39, May, 1981 (in Japanese).

10) Sakai, F. and Nagai, M. : A proposal for intermediate diaphragm design in curved steel box girder bridges, Proc. of JSCE, No. 305, pp. 11 22, January, 1981 (in Japanese).

11) Usuki, T. : An analysis of multi-cell box girder bridges with varied cross section under consideration of cross sectional distortion, Proc. of JSCE, No. 368/I-5, pp. 265 274, April, 1986 (in Japanese).

12) Takahashi, K., Yonago, A. and Mizuno, M. : Distortion of curved thin-walled open-cross-section members, Proc. of JSCE, No. 320, pp. 47 55, April, 1982 (in Japanese).

13) Hikosaka, H. and Takami, K. : Formulation of distortional behavior of thin-walled curved beam with open cross section, Proc. of JSCE, Structural Eng. /Earthquake Eng., I-3, pp. 91 100, April, 1985.

14) Beyer, K. : Statik im Stahlbetonbau, Springer-Verlag, pp. 756 757, 1956.

15) Sedlacek, G. : Systematische Darstellung des Biege- und Verdrehvorganges für prismatische Stäbe mit dünnwandigem Querschnitt unter Berücksichtigung der Profilverformung, Fortschrittbericht, VDI-Z, Reihe 4, Heft 8, VDI-Verlag, 1968.

16) Usuki, T. : Ein Beitrag zur Theorie dünnwandiger prismatischer Stäbe mit offen-geschlossenem Profil aus vier Scheiben, Die Bautechnik, Vol.60, Heft 1, pp. 14 23, 1983.

(Received November 11 1986) 